Int. J. Dev. Biol. 52: 397-405 (2008)

doi: $10.1387 / \mathrm{ijdb} .072424 \mathrm{fk}$

\title{
Expression of protocadherin 18 in the CNS and pharyngeal arches of zebrafish embryos
}

\author{
FUMITAKA KUBOTA, TOHRU MURAKAMI*, YUKI TAJIKA and HIROSHI YORIFUJI \\ Neuromuscular and Developmental Anatomy, Gunma University Graduate School of Medicine, Gunma, Japan
}

\begin{abstract}
Here, we report the results of molecular cloning and expression analyses of a nonclustered protocadherin (pcdh), pcdh18 in zebrafish embryos. The predicted zebrafish pcdh18 protein shows $65-66 \%$ identity and $78-79 \%$ homology with its mammalian and Xenopus counterparts. It has a Disabled-1 binding motif in its cytoplasmic domain, which is characteristic of pcdh18. Zebrafish embryos expressed pcdh 18 by the early gastrula stage, $6 \mathrm{~h}$ post-fertilization (hpf), in their animal cap but not in the germ ring or the shield. pcdh 18 was expressed in the neural tube and the central nervous system (CNS) from $12 \mathrm{hpf}$. Some populations of cells in the lateral neural tube and spinal cord of 12-18 hpf embryos expressed pcdh 18, but expression in these cells disappeared by $24 \mathrm{hpf}$. The hindbrain of embryos at 24-56 hpf expressed pcdh18 in cells closely adjacent to the rostral and caudal rhombomeric boundaries in a thread-like pattern running in the dorsoventral direction. The pcdh 18-positive cells were localized in the ventral part of the hindbrain at $24 \mathrm{hpf}$ and in the dorsal part from $36 \mathrm{hpf}$. pcdh18 was also expressed in the telencephalon, diencephalon, tectum, upper rhombic lip, retina and otic vesicle. Expression in the CNS decreased markedly before hatching. Pharyngeal arch primordia, arches, jaws and gills expressed pcdh 18, and the molecule was also expressed in some endodermal cells in late embryos.
\end{abstract}

KEY WORDS: Danio rerio, central nervous system, pharyngeal arch, protocadherin, rhombomere

Differential adhesion plays a pivotal role in morphogenesis during embryonic development (Steinberg, 1970). The cadherin superfamily is thought to provide a major molecular basis for such cell adhesion (for review, Halbleib and Nelson, 2006; Takahashi et al., 2005). Protocadherins (pcdh) are the largest subfamily of cadherins, which have common structural features in the extracellular domains, but no extensive similarities in their cytoplasmic domain unlike classic cadherins. Many of pcdhform gene clusters analogous to the immunoglobulin clusters. These clustered pcdh are more or less differentially expressed in the central nervous system (CNS), and have been suggested to be involved in systematic control of morphogenesis of CNS (Bass et al., 2007, Esumi et al., 2005, Hirayama and Yagi, 2006, Tada et al., 2004). Howerver, recent findings indicate allelic and conbinational gene regulation for clustered pcdh (Morishita and Yagi, 2007). This makes non-clustered pchdmore attractive as a diversely controled adhesion machinary functioning for elaborate morphogenesis.

A few of non-clustered pcdh, namely pcdh8, 10, and 15, are known to play roles in particular morphogenic events (Kazmierczak et al., 2007, Le Guedard et al., 2007, Murakami et al., 2006, Rhee et al., 2003, Yamamoto et al., 1998), although the biological significance of most non-clustered $p c d h$ is unknown. A subclass of non-clustered pcdhincluding pchd1, 7, 8, 9, 10, 11, 17, 18and 19 is characterized by a shared and highly conserved cytoplasmic motif of unknown function, CM-2 (Wolverton and Lalande, 2001). In the course of our investigation of this pcdhsubclass, we cloned zebrafish $p c d h 18$ and found its interesting expression pattern in developing embryos.

\section{Cloning of pcdh18}

Zebrafish pcdh18cDNA was amplified by PCR from a cDNA library using primers designed from a zebrafish predicted gene homologous to known pcdh18 (Supplimentary Fig. S1). The deduced sequence of zebrafish pcdh18 consists of 1,150 amino

Abbreviations used in this paper: CNS, central nervous system; Dab1, Disabled1; DNA, deoxyribonucleic acid; dpf, days post-fertilization; hpf, hours postfertilization; ISH, in situ hybridization; krx-20, krox-20; pcdh, protocadherin; PBS, phosphate-buffered saline; PCR, polymerase chain reaction; PTU, 1phenyl-2-thiourea; RNA, ribonucleic acid.

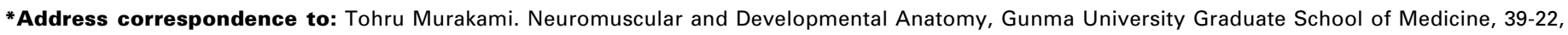
Showa-machi 3-chome, Maebashi, Gunma 371-8511, Japan. Fax: 81+27-220-7916. e-mail: tohru.murakami@gunma-u.ac.jp
} 

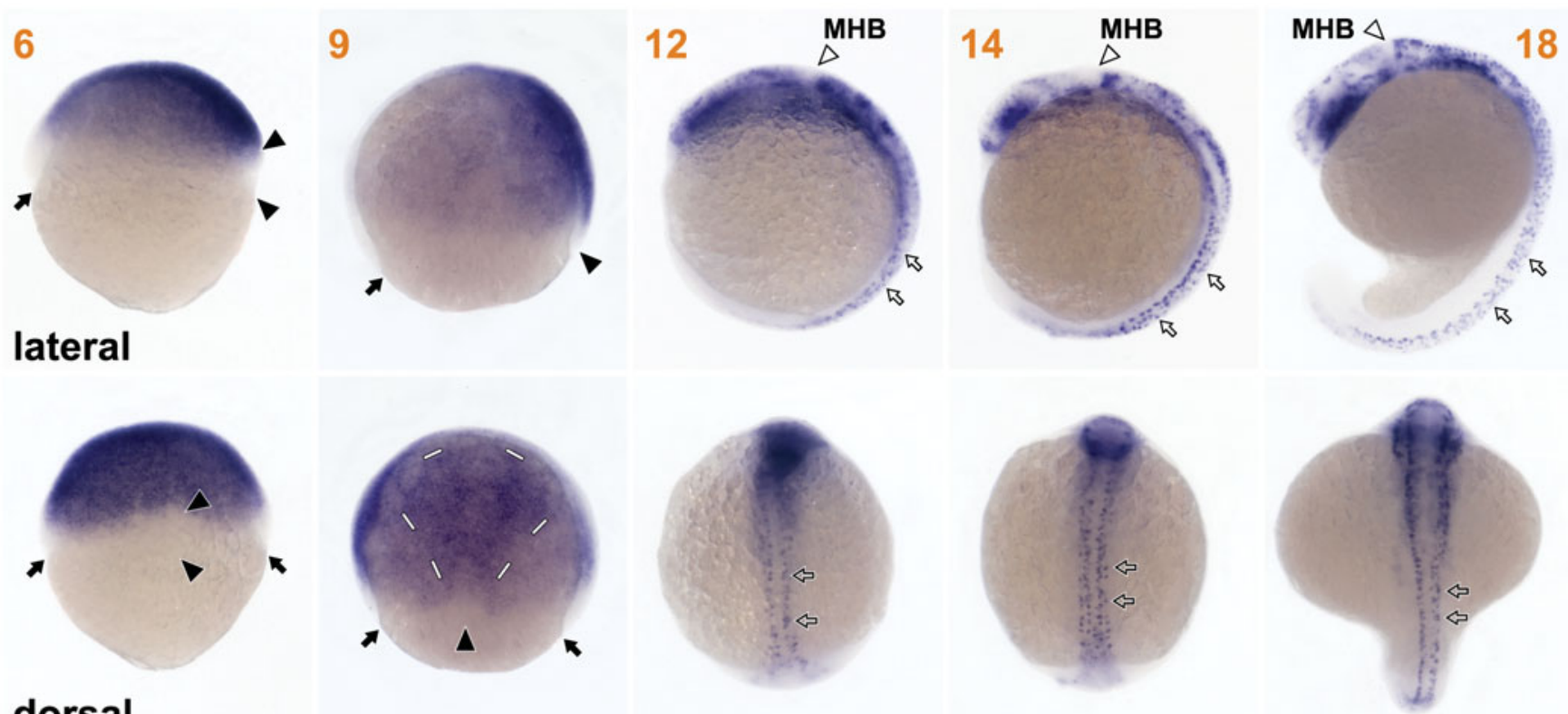

\section{dorsal}
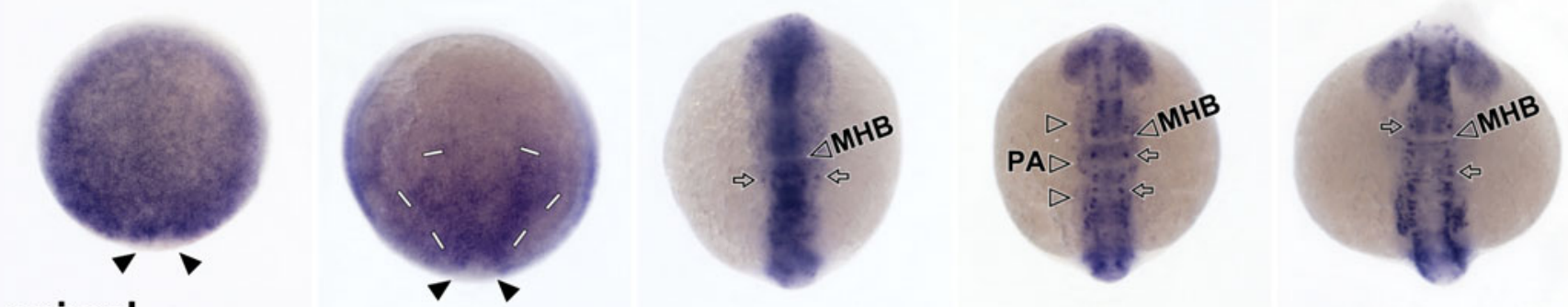

\section{animal}
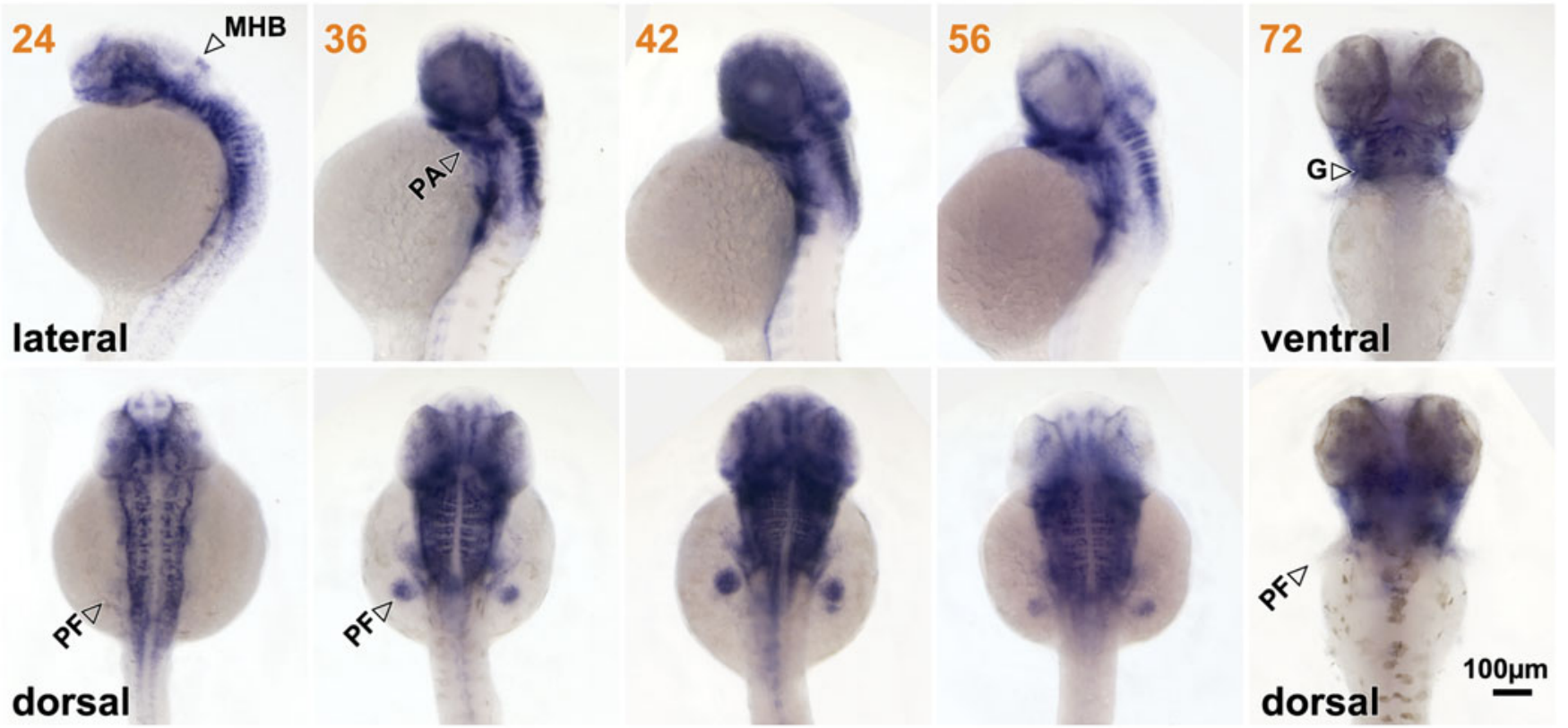

Fig. 1. Overview of pcdh18 expression in zebrafish embryos. Panels show extended depth of field photographs (see Experimental Procedures) of embryos stained blue by in situ hybridization forpcdh18 transcripts. Orange numbers indicate hours post-fertilization. Black arrows indicate the germ ring. Black arrowheads indicate the shield. Thin white bars show the triangular area of expression in 9 hpf embryos. Open arrows indicate punctuate expression in the head and trunk. The animal pole is aligned toward the top in lateral, dorsal, and ventral views. Dorsal is to the right in lateral views and down in animal pole views. Abbreviations: G, gills; MHB, midbrain-hindbrain boundary; PA, pharyngeal arch primordia or arches; PF, pectoral fin. 
acids, and shows conservation of characteristic features of the pcdh family, including a signal sequence, six cadherin domains, a single transmembrane domain, and a cytoplasmic domain (Supplementary Fig. S2). It shows 65-66\% identity and 78-79\% homology at the amino acid level with known pcdh18 and KIAA1562 proteins of human, mouse, rat, and Xenopus. The first five cadherin domains and the transmembrane domain show the highest degrees of identity and homology (Supplementary Fig. S3). Zebrafish pcdh18 has a Disabled-1 (Dab1) binding motif (F/ YxNP, or Phe/Tyr-any-Asn-Pro) in its cytoplasmic domain, which is characteristic of pcdh18 of different species (Homayouni et al., 2001) (Supplementary Fig. S2). Dab1 is an adapter protein, which mediates Reelin signals that affect cell migration and positioning in mammalian brain development. Dab1 has been shown to interact with pcdh18 viathe binding motif. Zebrafish pcdh18 has a CM-2 motif in the cytoplasmic domain.

\section{pcdh18 expression in early embryos}

The transcript of pcdh18 was first expressed diffusely in the animal cap of early gastrula embryos at 6 hours post-fertilization (hpf) (Fig. 1, 6 hpf). The expression was somewhat more intense dorsally than ventrally and was excluded from the germ ring and the shield (dorsal organizer). By the mid-gastrula stage or $9 \mathrm{hpf}$, pcdh18became more obviously localized to the dorsal half of the embryos. A roughly triangular pattern of expression appeared in the prospective head region (Fig. 1, $9 \mathrm{hpf}$ ), and the head structures continued to express pcdh18throughout development (Fig. 1, 9-72 hpf).

By $12 \mathrm{hpf}$, small punctuate expression appeared in the head and the trunk (Fig. 1, 12-14 hpf). The dots were roughly aligned on two or four parasagittal lines but not in precise symmetry. The punctuate expression began to decrease at $18 \mathrm{hpf}$ and had mostly disappeared by 24 hpf (Fig. 1, 18-24 hpf). Transverse cryostat sections revealed that the dots represented pcdh18-expressing cells in the lateral neural tube and spinal cord (Fig. 2, A-C). Based on their location (Bernhardt et al., 1990, Kuwada et al., 1990, Myers et al., 1986), these pcdh18-positive cells seemed to correspond to a subset of spinal interneurons, and the dorsolateral cells may be Rohon-Beard neurons. Further studies to determine their identity are currently underway in our laboratory.

\section{Expression in the brain}

In 12-hpf embryos, pcdh18 was expressed diffusely in the rostral region (Fig. 1, $12 \mathrm{hpf}$ ). By $14 \mathrm{hpf}$, the expression became more defined to structures such as eye buds, forebrain, midbrain, hindbrain, and pharyngeal arch primordia (Fig. 1, 14 hpf). In 18-
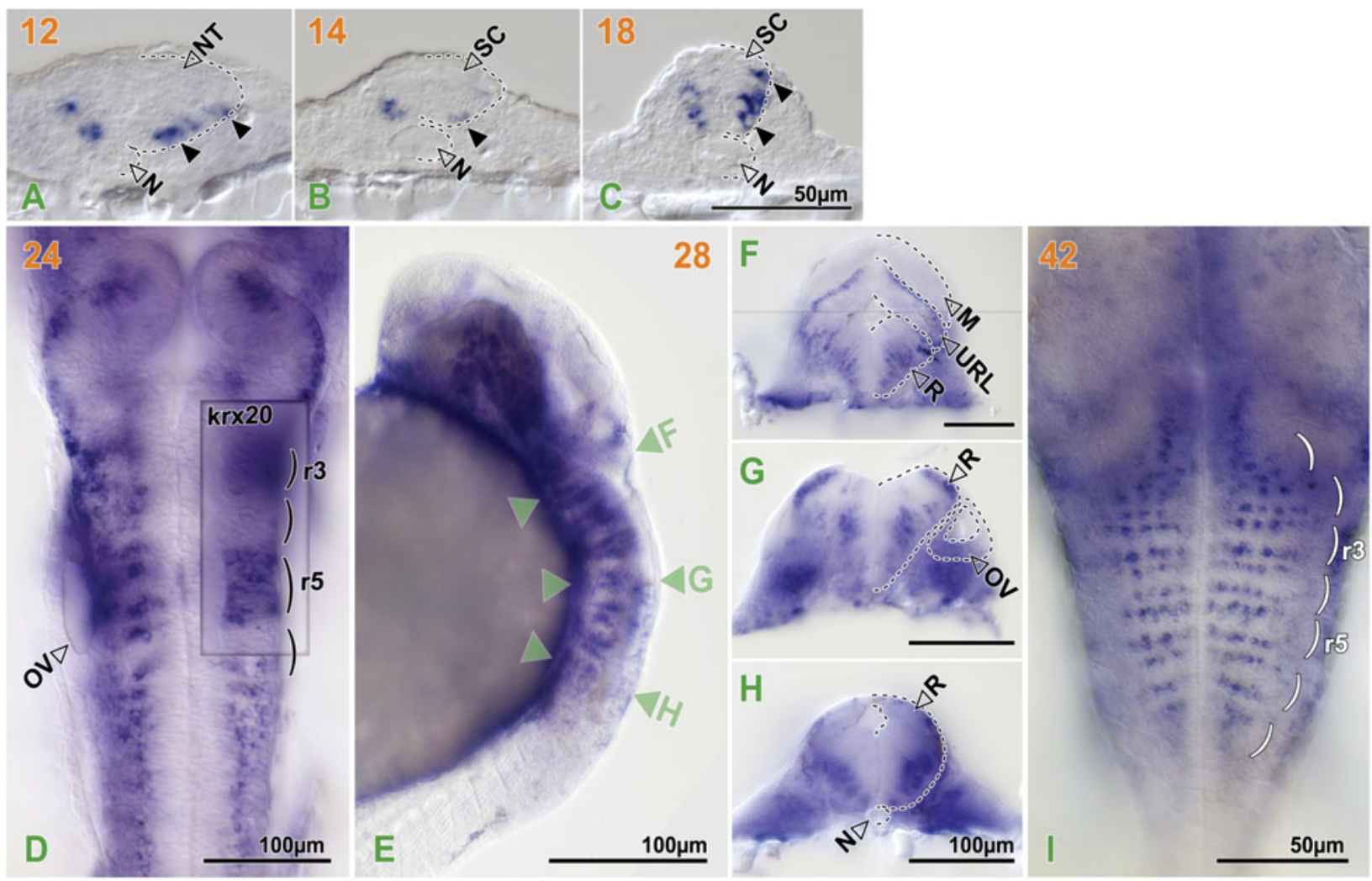

Fig. 2. pcdh18 expression in the hindbrain and spinal cord. Orange numbers indicate hours post-fertilization. Black dotted lines outline the structures indicated. Black arrowheads in transverse sections of the trunk of 12-18 hpf embryos (A-C) (dorsal to top) indicate pcdh18-expressing cells in the lateral neural tube and spinal cord. (D) Dorsal view of a 24 hpf embryo stained for pchd18 and krx-20 with single-color double in situ hybridization. Parentheses indicate rhombomeres. Inset shows a focal plane of an area of krx-20 expression which is located ventral to that of pcdh 18 expression. Green arrowheads in the lateral view of a 28-hpf embryo (E) indicate focal planes in hand-cut sections (F-H) (dorsal to top) made from the very same embryo. (F) A composite picture of two closely adjacent focal planes. (I) A dorsal view of the hindbrain (rostral to the top) of a 42 hpf embryo with patterns of transverse dotted lines. Abbreviations: $M$, midbrain; $N$, notochord; NT, neural tube; OV, otic vesicle; $R$, rhombencephalon; 3 \& 5 , rhombomere 3 \& 5; SC, spinal cord; URL, upper rhombic lip. 


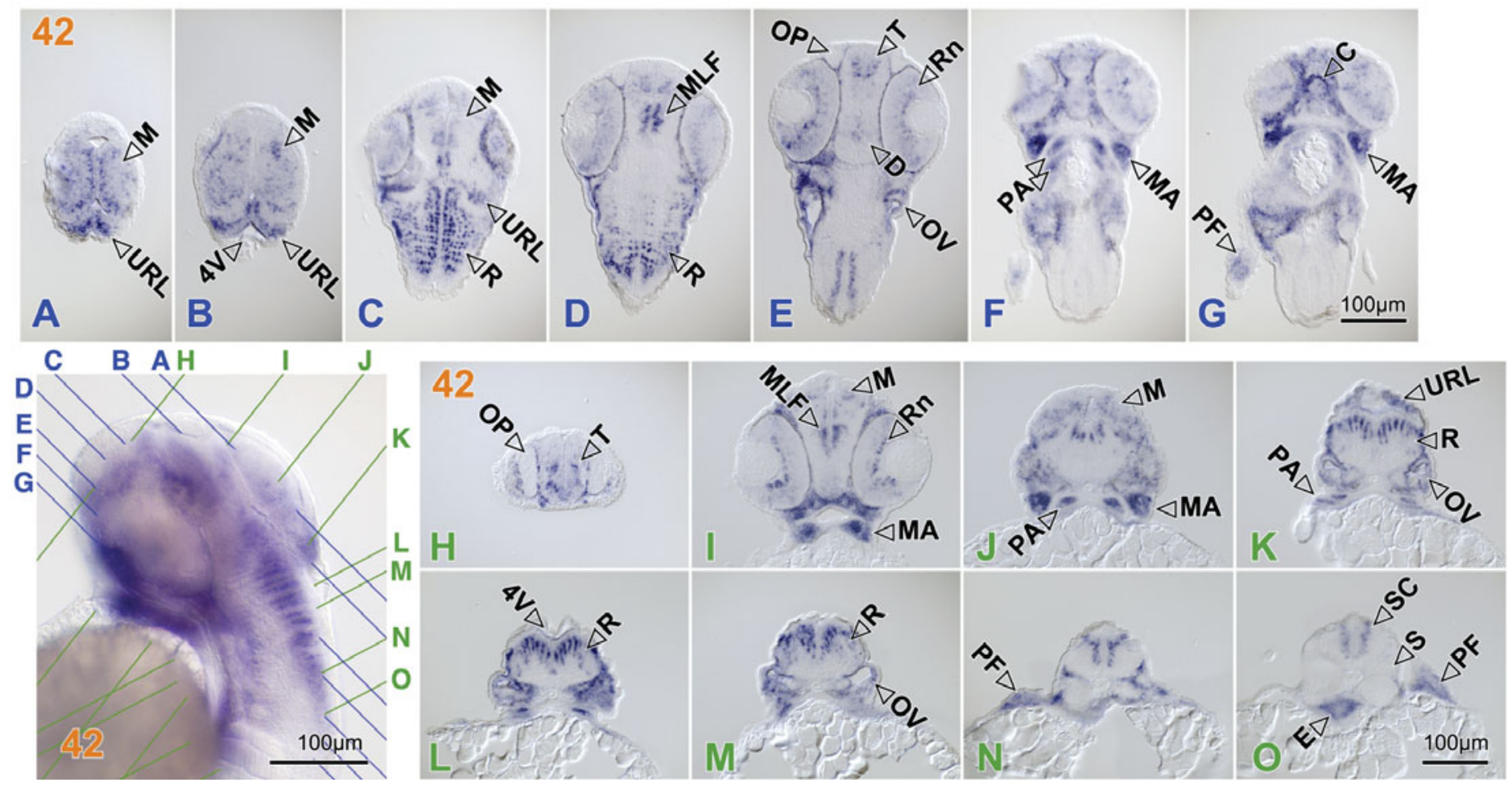

Fig. 3. Expression in the CNS and pharyngeal arches in sections. Orange numbers indicate hours post-fertilization. Colored lines $A-O$ in the lateral view of a 42-hpf embryo (lower left) indicate planes of serial sections (A-G) (horizontal sections; rostral to the top) and (H-O) (transverse sections; dorsal to the top). Abbreviations: $4 \mathrm{~V}$, forth ventricle; $C$, chin; $D$, diencephalon; $E$, endoderm; $M$, midbrain; MLF, medial longitudinal fascicle; OV, otic vesicle; $O P$, olfactory pit; PA, pharyngeal arches; PF, pectoral fin; $R$, rhombencephalon; $R n$, retina; $S$, somite; $T$, telencephalon; URL, upper rhombic lip.

hpf embryos, pcdh18 expression continued in the eye buds, otic vesicles, forebrain, and ventral parts of the midbrain through hindbrain (Fig. 1, $18 \mathrm{hpf}$ ). There was a gap of expression at the midbrain-hindbrain boundary during the period from 14 to $18 \mathrm{hpf}$ (Fig. 1, 14-18 hpf).

By $24 \mathrm{hpf}$, transverse lines of expression appeared in dorsal and lateral aspects of the rhombencephalon (Fig. 1, 24-56 hpf). The pattern prompted us to identify the alignment of the lines to rhombomeres in terms of the rhombomere segmentation. Double staining for pcdh18 and krox-20 (Oxtoby and Jowett, 1993) transcripts showed that the lines were localized closely adjacent to the rostral and caudal rhombomeric boundaries (Fig. 2, D). Examination of transverse hand-cut sections from a whole-mount stained 28-hpf embryos revealed that the expression was localized in the ventral part of rhombencephalon running in transverse planes from ventrolateral to dorsomedial (Fig. 2, E-H).

During the period from 36 to $56 \mathrm{hpf}$, pcdh 18 continued to be expressed in the rhombencephalon in transverse lines adjacent to the rhombomere boundaries (Fig. 1, 42-56 hpf; Fig. 2, I). Unlike earlier embryos, pchd18 was expressed in the dorsal part of the rhombencephalon from 36 hpf (Fig. 1, 36-56 hpf; Fig. 3, K-N). Examination of transverse and coronary sections showed that the staining pattern was actually threads of pcdh18-positive cells running dorsoventrally in transverse planes (Fig. 3), similar to the radial glia (Trevarrow et al., 1990). Some signaling and transcription factors such as deltaA (Delta-Notch signaling), rasgef1b (Ras GEF), and beta3.1 (bHLH), have been reported to show similar expression patterns in the hindbrain (Adolf et al., 2004, Epting et al., 2007, Riley et al., 2004). Although functional relationships between these signals and pchd18 remain to be elucidated, it is interesting to assume that pcdh18 is involved in rhombomeric boundary formation driven by Delta-Notch signaling.

Analysis of sections also showed that pcdh18was expressed in the telencephalon, medial longitudinal fascicle of the diencephalon, tectum, and upper rhombic lip. pcdh18was expressed in sensory systems such as the retina and otic vesicles, but not in olfactory pits or lateral lines (Fig. 3, A-O). The expression in the retina was most intense in the innermost ganglion cell layer. The expression in the CNS decreased by 56 hpf (Fig. 1, 56 hpf). The distinct pattern in the hindbrain was almost completely abolished by 72 hpf (Fig. 1, 72 hpf).

\section{Expression in pharyngeal arches and other structures}

pcdh 18 was expressed in the pharyngeal arch primordia by 14 hpf (Fig. 1, 14 hpf). It was expressed in pharyngeal, maxillary, and mandibular arches in later embryos (Fig. 3, F, G, I, J), and in the branchial and jaw arches at $72 \mathrm{hpf}$ (Fig. 1, $72 \mathrm{hpf}$, and Supplimentary Fig. S4, A). Cryostat sections of 72 hpf embryos revieled that pch18 was expressed in the cells encaplulating the branchial cartilages (Supplimentary Fig. S4, A, C). Pectral fin buds expressed pcdh18at $24 \mathrm{hpf}$ through $56 \mathrm{hpf}$ (Fig. 1, 24-56 hpf). Some endodermal cells expressed pcdh18 in late embryos (Fig. 3, O).

Previous studies indicated the involvement of pcdh18 protein and reelin-Dab1 signaling in mammalian odontogenesis (Carroll et al., 2001, Fukasawa et al., 2005, Heymann et al., 2001, Homayouni et al., 2001). Zebrafish has pharyngeal teeth which 
attach to the 5th branchial arch at 4-6 days post fertilization (dpf). Since zebrafish pcdh18 share Dab1 binding site, we expected to see some increase of pcdh18 expression in, or related to the teeth. In contrast to the expectations, we could not find toothrelated pchd18 expression at $72 \mathrm{hpf}$ nor $5 \mathrm{dpf}$ (Supplementary Fig. S4).

\section{Conclusions}

We cloned a zebrafish non-clustered pcdh, pcdh18, and characterized its embryonic expressions in detail. pcdh18 was expressed in the neural tube, CNS, eyes, otocysts, pharyngeal arches, and some endodermal cells. The expression in the hindbrain was particularly interesting in terms of the rhombomere segmentation. We are addressing the identities of the cells expressing $p c h d 18$, and the functional significances of $p c d h 18 \mathrm{in}$ the CNS morphogenesis.

The expression pattern of $p c d h 18$ in zebrafish embryos seemed to be quite different from that in mice; pcdh18expression is rather systemic in rodents (Homayouni et al., 2001, Kim et al., 2007), whereas it was limited to the CNS and head structures in zebrafish. Teleost fish including zebrafish often have diversified alleles homologous to a mammalian counterpart supposedly due to ancient whole gene duplications (Stock, 2007, Wittbrodt et al., 1998). Blast searches indicate that there are two alleles (EU267178 and our AB297803) in the zebrafish genome homologous to mammalian pcdh18. It is interesting to suppose these pcdh18 alleles acquired differential rolls in zebrafish development. Nonetheless, further comparative studies on the functional diversity of pcdh18 in different species are required.

\section{Experimental Procedures}

\section{Fish}

Zebrafish (AB line) were obtained from the Zebrafish International Resource Center (Eugene, OR; http://zebrafish.org/zirc/). Fish embryos were raised at a standard temperature of $28.5^{\circ} \mathrm{C}$ (Westerfield, 2000), fixed in $4 \%$ paraformaldehyde - PBS at various time points, and stored in methanol at $-70^{\circ} \mathrm{C}$ until use for expression analyses. PTU (1-phenyl-2thiourea) at $0.003 \%$ was used for older embryos to suppress pigmentation.

\section{Cloning of zebrafish pcdh18}

Zebrafish pcdh18 cDNA was amplified by PCR from a zebrafish embryonic cDNA Uni-ZAP XR library (discontinued; Stratagene, La Jolla, CA) using primers 5'-GGA TTT ATC ATG CTT TAA TCT ACA CCG AAC3' and 5'-GGA TAA TTA GCA TTC ACA AAA CAC AAT ACA TCC-3', designed to flank the coding region of a zebrafish predicted protein similar to pcdh18 (accession: XM_685424). The PCR product was cloned in the pCR-Blunt II vector using a ZeroBlunt TOPO PCR Cloning Kit (Invitrogen Japan, Tokyo, Japan). The pcdh18cDNA was sequenced using an ABI Prism 3100 (Applied Biosystems Japan, Tokyo, Japan) (accession: AB297803). Pair-wise alignment and multiple alignment analyses were performed using DNA Strider 1.4 (C. Marck, CEA, Cedex, France) and ClustalW (EMBL-EBI, http://www.ebi.ac.uk/clustalw/), respectively.

\section{In situ hybridization}

RNA probes for in situ hybridization (ISH) were synthesized using a DIG RNA Labeling Kit (Roche Diagnostics K.K., Tokyo, Japan). Embryos were stained by whole-mount ISH as described previously (Bellipanni et al., 2000, Kubota et al., 2007, Murakami et al., 2006). Single-color double ISH for pcdh18 and krx-20 was done for 24-hpf embryos to identify the alignment of $p c h d 18$ staining to the rombomeres. Two-color double ISH was not successful for us, supposedly due to diminishing expression of krx-20 at $24 \mathrm{hpf}$.

\section{Microscopy}

Stained whole embryos, embryos with the yolk removed, and hand-cut sections made from stained embryos using rasor blades were used for microscopy. A whole-mount embryo was held stationary in the pit of an agarose plate cast with glass beads. For serial cryostat sections, stained embryos were cryoprotected in $20 \%$ sucrose-PBS, immersed in O.C.T. Compound (Sakura Finetek, Tokyo, Japan), and snap-frozen in liquid nigrogen. Nikon AZ-100 and E-1000 compound microscopes were used for microscopic analyses. Photographs were taken using Olympus E-330 and E-3 digital SLR cameras in RAW format and processed with Adobe Lightroom. For extended depth of field pictures of whole-mount specimens, a series of photographs were taken with shifting focus throughout the embryo and processed with the "Extended Depth of Field" plug-in (Forster et al., 2004) (http://bigwww.epfl.ch/demo/edf/) for ImageJ software (http://rsb.info.nih.gov/ij/). Adobe Photoshop was used for adjustments, including color temperature corrections to make the background a neutral gray, automatic black and white level correction, and gamma adjustment to 1.25 for the photographs of whole-mount and hand-cut sections to allow details to be seen clearly in darkly stained areas in prints.

\section{Acknowledgments}

This work was supported by Grants-in-Aid awarded to T.M. from the Ministry of Education, Culture, Sports, Science, and Technology of Japan (MEXT) and the Initiative for Attractive Education in Graduate Schools awarded to Gunma University from MEXT.

\section{References}

ADOLF, B., BELLIPANNI, G., HUBER, V. and BALLY-CUIF, L. (2004). atoh1.2 and beta3.1 are two new bHLH-encoding genes expressed in selective precursor cells of the zebrafish anterior hindbrain. Gene Expr Patterns 5: 35-41.

BASS, T., EBERT, M., HAMMERSCHMIDT, M. and FRANK, M. (2007). Differential expression of four protocadherin alpha and gamma clusters in the developing and adult zebrafish: DrPcdh2gamma but not DrPcdh1gamma is expressed in neuronal precursor cells, ependymal cells and non-neural epithelia. Dev Genes Evo/217: 337-51.

BELliPANNI, G., MURAKAMI, T., DOERRE, O.G., ANDERMANN, P. and WEINBERG, E.S. (2000). Expression of Otx homeodomain proteins induces cell aggregation in developing zebrafish embryos. Dev Bio/223: 339-53.

BERNHARDT, R.R., CHITNIS, A.B., LINDAMER, L. and KUWADA, J.Y. (1990). Identification of spinal neurons in the embryonic and larval zebrafish. J Comp Neuro/302: 603-16.

CARROLl, P., GAYET, O., FEUILLET, C., KALLENBACH, S., DE BOVIS, B. DUDLEY, K. and ALONSO, S. (2001). Juxtaposition of CNR protocadherins and reelin expression in the developing spinal cord. Mol Cel/ Neurosci17: 611-23.

EPTING, D., VORWERK, S., HAGEMAN, A. and MEYER, D. (2007). Expression of rasgef1b in zebrafish. Gene Expr Patterns 7: 389-95.

ESUMI, S., KAKAZU, N., TAGUCHI, Y., HIRAYAMA, T., SASAKI, A., HIRABAYASHI, T., KOIDE, T., KITSUKAWA, T., HAMADA, S. and YAGI, T. (2005). Monoallelic yet combinatorial expression of variable exons of the protocadherin-alpha gene cluster in single neurons. Nat Genet 37: 171-6

FORSTER, B., VAN DE VILLE, D., BERENT, J., SAGE, D. and UNSER, M. (2004). Complex wavelets for extended depth-of-field: a new method for the fusion of multichannel microscopy images. Microsc Res Tech 65: 33-42.

FUKASAWA, K., SAHARA, N., MORIYAMA, K., KUNO, T., FUJII, S., OTOGOTO, J.I., OTA, N., UDAGAWA, N., YAGASAKI, H. and OZAWA, H. (2005). Identification of protocadherin 18-like protein in horse molar cementum. Journal of the Matsumoto Dental University Society 31: 160-166.

HALBLEIB, J.M. and NELSON, W.J. (2006). Cadherins in development: cell adhesion, sorting, and tissue morphogenesis. Genes Dev20: 3199-214.

HEYMANN, R., KALLENBACH, S., ALONSO, S., CARROLL, P. and MITSIADIS, 
T.A. (2001). Dynamic expression patterns of the new protocadherin families CNRs and Pcdh-gamma during mouse odontogenesis: comparison with reelin expression. Mech Dev 106: 181-4.

HIRAYAMA, T. and YAGI, T. (2006). The role and expression of the protocadherinalpha clusters in the CNS. Curr Opin Neurobio/16: 336-42.

HOMAYOUNI, R., RICE, D.S. and CURRAN, T. (2001). Disabled-1 interacts with a novel developmentally regulated protocadherin. Biochem Biophys Res Commun 289: 539-47.

KAZMIERCZAK, P., SAKAGUCHI, H., TOKITA, J., WILSON-KUBALEK, E.M., MILLIGAN, R.A., MULLER, U. and KACHAR, B. (2007). Cadherin 23 and protocadherin 15 interact to form tip-link filaments in sensory hair cells. Nature 449: 87-91.

KIM, S.Y., CHUNG, H.S., SUN, W. and KIM, H. (2007). Spatiotemporal expression pattern of non-clustered protocadherin family members in the developing rat brain. Neuroscience 147: 996-1021.

KUBOTA, F., MURAKAMI, T., MOGI, K. and YORIFUJI, H. (2007). Cadherin-6 is required for zebrafish nephrogenesis during early development. Int J Dev Biol 51: 123-9.

KUWADA, J.Y., BERNHARDT, R.R. and NGUYEN, N. (1990). Development of spinal neurons and tracts in the zebrafish embryo. J Comp Neuro/302: 617-28.

LE GUEDARD, S., FAUGERE, V., MALCOLM, S., CLAUSTRES, M. and ROUX, A.F. (2007). Large genomic rearrangements within the PCDH15 gene are a significant cause of USH1F syndrome. Mol Vis 13: 102-7.

MORISHITA, H. and YAGI, T. (2007). Protocadherin family: diversity, structure, and function. Curr Opin Cell Biol 19: 584-92.

MURAKAMI, T., HIJIKATA, T., MATSUKAWA, M., ISHIKAWA, H. and YORIFUJI, H. (2006). Zebrafish protocadherin 10 is involved in paraxial mesoderm development and somitogenesis. Dev Dyn 235: 506-14.

MYERS, P.Z., EISEN, J.S. and WESTERFIELD, M. (1986). Development and axonal outgrowth of identified motoneurons in the zebrafish. JNeurosci6: 227889.

OXTOBY, E. and JOWETT, T. (1993). Cloning of the zebrafish krox-20 gene (krx20) and its expression during hindbrain development. Nucleic Acids Res 21:
1087-95.

RHEE, J., TAKAHASHI, Y., SAGA, Y., WILSON-RAWLS, J. and RAWLS, A. (2003). The protocadherin papc is involved in the organization of the epithelium along the segmental border during mouse somitogenesis. Dev Bio/254: 248-61.

RILEY, B.B., CHIANG, M.Y., STORCH, E.M., HECK, R., BUCKLES, G.R. and LEKVEN, A.C. (2004). Rhombomere boundaries are Wnt signaling centers that regulate metameric patterning in the zebrafish hindbrain. Dev Dyn231:278-91.

STEINBERG, M.S. (1970). Does differential adhesion govern self-assembly processes in histogenesis? Equilibrium configurations and the emergence of a hierarchy among populations of embryonic cells. J Exp Zoo/173: 395-433.

STOCK, D.W. (2007). Zebrafish dentition in comparative context. Journal of Experimental Zoology Part B: Molecular and Developmental Evolution 308B: 523-549.

TADA, M.N., SENZAKI, K., TAI, Y., MORISHITA, H., TANAKA, Y.Z., MURATA, Y., ISHII, Y., ASAKAWA, S., SHIMIZU, N., SUGINO, H. et al. (2004). Genomic organization and transcripts of the zebrafish Protocadherin genes. Gene 340: 197-211.

TAKAHASHI, Y. (2005) Common mechanisms for boundary formation in somitogenesis and brain development: shaping the 'chic' chick. Int. J. Dev. Biol. 49: 221-230.

TREVARROW, B., MARKS, D.L. and KIMMEL, C.B. (1990). Organization of hindbrain segments in the zebrafish embryo. Neuron 4: 669-79.

WESTERFIELD, M. (2000). The Zebrafish Book: A Guide for the Laboratory Use of Zebrafish (Danio rerio). University of Oregon Press, Eugene, OR, USA.

WITTBRODT, J., MEYER, A. and SCHARTL, M. (1998). More genes in fish? Bioessays 20: 511-515.

WOLVERTON, T. and LALANDE, M. (2001). Identification and characterization of three members of a novel subclass of protocadherins. Genomics 76: 66-72.

YAMAMOTO, A., AMACHER, S.L., KIM, S.H., GEISSERT, D., KIMMEL, C.B. and DE ROBERTIS, E.M. (1998). Zebrafish paraxial protocadherin is a downstream target of spadetail involved in morphogenesis of gastrula mesoderm. Development 125: 3389-97. 


\section{Supplementary Material}

\footnotetext{
1 ggatttatca tgctttaatc tacaccgaac aatccaccag ccagtcttct gtgtgtgtgg 61 aataacaact tcgcgcacgt cttcaaatAT GGGAACAACT AAGCACTCCA GCGACTGGAA 121 TGTTTTGTTT TGCAAGACGC TTCTGAAACT TATGCTTCTG GCGGCCGTGG CGCACAATGT 181 TTCGGGGAA ACTTTAAAAT ACAAAGTTCT GGAAGAGCAA AgAgTTGgGA CGgTAATCGC 241 CAgGCtgAAA GAgGATGtAg CCGGTCTGTT AgCCAAACTC CCGAGCTCCG TGTCTCCGCG 301 CTTCAGAGCG ATGCAGAgAg GCAGCACCCC TCTGCTCTCC GTGCGCGAGg AgGACGGAGA 361 GATCAGCATA GCCACCAAAA TCGACCGAGA AAAGCTTTGT GAGAAAAACT TAAACTGCAC 421 TATCGAGTTC GACGTGATTA CTATTCCCAC GGAATACCTC CAACTGTTTC ACATTCAAGT 481 GGAAGTGTTG GACATCAACG ACAACGCTCC GCAGTTTTCG CGCGCCATCA TCCCTATAGA 541 GATATCCGAG AgCGCGTCTG TGgGCACACG CTTCCCTCTG GATAgCGCGg TAGACCCGGA 601 TGTCGGGGAA AACGCGCTGC ACACCTACTC GCTCACTCGg AATAACTTTT TCAAAATCGA 661 TATCAGGACG CGAACGGACG GCGCAAAGTA CGCGGACCTT GTTGTAATGA GAGAACTTGA 721 CAGGGAAACA CAGTCCAGTT ATCAACTACA ACTCACAGCT TCAGACTCTG GCGTACCGCC 781 GAAATCTGGC TCAACTCTGC TTAAAATCAG CATATCTGAT TCCAACGATA ATAGCCCGGC 841 GTTTGACGAG CAGGCTTACG TGGTCAACCT CCTGGAAAAT TCATCACTGG AGACGCTCTT 901 GGTAGACTTG AACGCTACAG ACCCGGATGA AGGCAATAAT GGCAAAATCG TGTATTCTTT 961 CAGCAGCCAC GTGCCCCCCA AAATCCTAGA GACTTTCAAA ATTAACCCAG ATAATGGCCA

1021 CATCACTCTT ATTAAGAAAG TTGACTACGA AACCACGTCT TCATATGAAA TAGATGTGCA 1081 GGCTCAGGAT ATGGGTCCCA ACTCCATTCC AGGCCACTGT AAAATAATAA TCAAAGTGGT 1141 CGACGTCAAC GACAACAAGC CAAAAATCAA CATCAACCTC ATGACTCAGG GCAAAGAGGA 1201 GGTGGCCTAC ATCTCAGAGG CCTCCCCTGT TGATACCTTC ATCGCATTAG TGCGAGTGGA 1261 TGACAGTGAC TCGGGTATAA ACGGTGAGGT TGTGTGCCGT CTTCATGGCC ATGGGCATTT 1321 TCGCCTGCAG AAGACCTACG AGAACAACTA CATGATTCTC ACAAATGTGT CTCTGGACCG 1381 TGAGAAACGC TCTGAATATA GCTTGACGGT GATCGCCGAA GACCGTGGCA CGCCAAGCCT 1441 ATCCACCATC AAACACTTCA CAGTGCAGGT GCAGGATGAA AATGACAACC CTCCCCGCTI 1501 TGAAAAGAGC CGATATGAAA TCTTCAAGTC TGAGAACAAT TCCCCTGGGG CATACCTGAC 1561 GTCAGTGGTG GCCACTGACC CAGATTTGGG GCCTAATGGA CAGGTGACCT ACTCAATTGT 1621 GGAAAGCCAG GTCCAGGGCA GCTCTATTTC AACTTATGTT ACTATTGACC CATCCAACGG 1681 GGCTGTGTAT GCACTCCGAA GCTTTGATCG GGAAGAAGTT GGCCGGCTTT CGTTCACCGT 1741 GCAGGCGCGG GATGGCGGAA GCCCAGCCTT TCTGAGCAGC AACACCACTG TGTTGCTCAC 1801 CATCCTAGAT GAAAATGACA ACCCTCCTAT TATTGTTGCC CCACTGCTAA GTAACCACAC 1861 AGCTGAGGTA CCATTATGGC GGCACGCCGA GTCTGGTCAT CTGGTGACAA TCATTAAGGC 1921 GACAGATCGT GATGCAGGAG CCAACAGCGA GCTGACTTGC TCCATAGTTG GAGGCAATGA 1981 CGAGGGACTC TTTGTCATTG ACCCACGCAG ATGTGAGTTA CGAACCAACG GAAGTGTGGA 2041 GGCCTCAGGA CGTGAGGGAT TTGATCTAGC CATCCTGGTG CAAGACAGA GTGCATCCAT 2101 TCGACTTTCA GCGAGGGCCG TCCTGCACAT CAGCCTACGG GACTATCCAG AAAGCTACTC 2161 CCTGAACCCT TCAGACATAA ACAACCAATC CCCACTAGAC GTGTCTATGA TCATCATCAT 2221 CTCCCTTGGT GCCATCTGTG GTGTGCTGCT CATTGTTATG GTAATGTTCG CAACCAGGTG 2281 CTCAAGAGA CAGAAGgACC CAAGGCACTC CTACAACTGC AGAGTGGCTG AATCCACCTA 2341 CCAAAACCAT CCCAAAAAAC CAGCCCGGCA GATCCACAAG GGTGACATCA CTCTGGTGCC 2401 CACAATTAAC GGGACCCTGC CTGTGAGAGC CCATCCTAGG TCCCCTTCAG CCTCCCCTGC 2461 TCCAGAAAGC CGGCAGAGCC ACCACAGCCG CCAATCACTC AACAGCCTGG TCACCATCTC 2521 ATCCAATCAT GTTCCAGAGA ACTTCGCCCT AGAACTTGCC CACGCCACAC CACCAGTCGA 2581 GGGCCAGTAC CAACCCAGAC CCAGTTTCCG TGGCAACAAA TACACCAGGA GCTACAGGTA 2641 TGCTTTGAAT GAGATGGACA AGTTCAGTCT GAAGGACAGT GGGCGAGGGG ATAGTGAGGC 2701 TGGAGACAGT GATTGTGAGA TGGGCAGAGA GTCTCCTCTG CTTGGAGAAG GCTTCAGTGA 2761 GCTCTTCACT CCTGATGGAC ATCACCGACT GCATCCCACG ATGAAGCTGT GCACTGAAGA 2821 GTGTCGAGTC CTGGGTCACT CTGACCAGTG CTGGATGCCT CCATTGCCAT CTCCAGCATC 2881 TTCTGATTAC AGAAGCAACC TTTATATCCC TGGAGAAGAC CCTCAGCAGA AAGCCACTCC 2941 TGAAGTGCCC CAATCTGGAG AGTCCACGCT GAGGAAAAAG AGTTTCTCCA CATTCGGCAA

3001 GGAGAGCGAG GAGGGAGAAG GAGAAGCAGA TGATGGAGAA GATCTATGTG GAACCACATC

3061 TTTGCTGTCA GAAATGAACA GCGTTTTTCA GAGGCTCCTT CCCTCCTCGC TGGATAATTA 3121 CAGCGAAACG AATGAAACTG AAAAGGCAAC CGCCTGTGCT GCAGGGGTGG GATCGCTGGA 3181 AAGAAGGAAG GGCCATTTAC CAGGTAAACC AAGCCCTGCT ACTTACCAGC AGAACGTTGC 3241 TGTTTGGGCA GCTAACACAC ACTTTCAAAA TCCCGGCCAT GGCCACGCCC CTGCTAGTCA 3301 CATGACAGCC CTGGTGGCTC CACTCCCCGC CCCTTTGCCT GCTCCAATGC CGGCATCAGC 3361 TTCCTGTTCA AAATGGCTGC CAGCAATGGA GGAAATCCCT GAAAATCACG AgGAGgACGA 3421 GCTGGAGTCT GTGCTCGGAC ACTTACACGG GAAGCGCTGC GATAGTCGCA GCGAAATTAT

3481 GGACGCCAGC GAGCTTGTTG CAGAAATCAA CAAACTCCTT CAAGATGTAA GgCAAAgCta

3541 aagattagac ttgtttttt tattattatt tatgagagtg gaaggtatg atatcgaaaa 3601 gagaagaaga aaaaaaaac acagagacct gcaattgtcg ttaaagttgc atttctggat 3661 gtattgtgtt ttgtgaatgc taattatcc
}

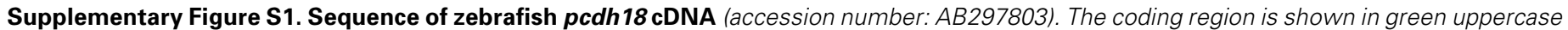
letters. 


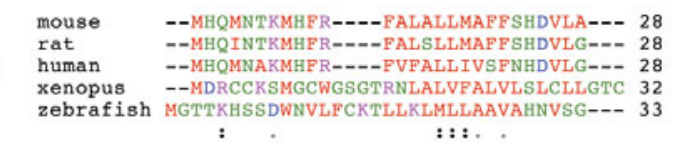

mouse KNLKYRIYEEQRVGSVIARLSEDVADVLLKLPNPSAVRFRAMPRGNSPLLVVNENTGEISIGAKIDREQLCOKNLNCSIEFDVLTLPTEHLQLFHIEVDVLDINDNSPQF 138 rat KNLKYIYEEQRVGSVIARLSEDVADVLLKLPNPSAIRFRAMPRGNSPLLVVNENTGEISIGAKIDREQLCQKNLNCSIEFDVLTLPTEHLQLFHIEVDVLDINDNSPQF 138 EC1 human KNLKYRIYEEQRVGSIARLSEDVADVLLKLPNPSTVRFRAMQRGNSPLLVVNEDNGEISIGATIDREQLCQKNLNCSIEFDVITLPTEHLQLFHIEVEVLDINDNSPQF 138 xenopus KNLKYRIYEEQMIGTVVARLSEDVADVLSKLPNP SSVRFRAMQRGSSPLLTVREDNGEISIGAKIDREQLC QRNSNCSIEFDVITLPTQHLQLFHIEVQVLDINDNAPQP 144

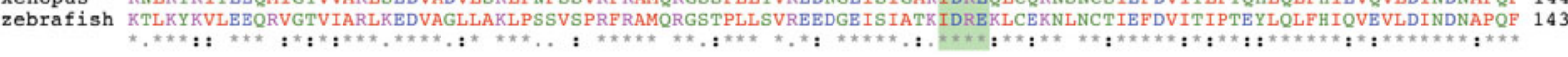

mouse SRPVIPIEISESAAVGTRIPLDSAFDPDVGENSLHTYSLSANDYFNIEVRTRTDGAKYAELIVVKELDRELKASYELQLTASDMGVPQRSGSSILKISISDSNDNSPAF 247 rat SRPVIPIEISESAAVGTRIPLDSAFDPDVGENSLHTYSLSANDYFNIEVRTRTDGAKYAELIVVKELDRELKASYELQLTASDMGVPQRSGSSILKISISDSNDNSPAF 247 EC2 human SRSLIPIEISESAAVGTRIPLDSAFDPDVGENSLHTYSLSANDFFNIEVRTRTDGAKYAELIVVRELDRELKSSYELQLTASDMGVPQRSGSSILKISISDSNDNSPAF 247 xenopus SRALIPIEISESAAVGTRIPLDSAFDPDVGDNSLFTYSLSPNAYFSIEVKTRTDGAKYABLVVIRELDRELQSSYELQLTAYDNGVPQRYGSSLLKISISDSNDNSPVF 25 zebrafish SRAIIPIEISESASVGTRPPLDSAVDPDVGENALHTYSLTRNNFFKIDIRTRTDGAKYADLVVMRELDRETQSSYQLQLTASDSGVPPKSGSTLLKISISDSNDNSPAF 252

mouse EQPSYTIQLLENSPVGTLLLDLNATDPDEGANGRIVYSFSSHVSPKIIETFKIDSEKGHLTLFKPVDYEITKSYEIDVQAQDLGPNSIPAHCKIIIKVVDVNDNKPEI 354 rat EOPSYTIOLLENSPVGTLLLDLNATDPDEGVNGRIVYSFSSHVSPKIIETFRIDSERGHLTLFKPVDYEITKSYEIDVOAODLGPNSIPAHCKIIIKVVDVNDNKPEI 354 ec3 human EQOSYIIOLLENSPVGTLLLDLNATDPDEGANGKIVYSFSSHVSPKIMETFKIDSERGHLTLFKOVDYEITKSYEIDVOAODLGPNSIPAHCKIIIKVVDVNDNKPEI 354 xenopus EKOSYVVOLPENSPLGTLLIDLNATDPDEGANGKVVYSFSSHVSPKITETFKIDPESGOLTLIKPVDYETTKSYEIDVOAODMGPNSIPAHCKIIINVVDVNDNKPEI 361 zebrafish DEQAYVVNLLENSSLETLLVDLNATDPDEGNNGKIVYSFSSHVPPKILETFKINPDNGHITLIKKVDYETTSSYEIDVQAQDMGPNSIPGHCKIIIKVVDVNDNKPKI 360

mouse SINLMSPGEEVSYVFEDPIDTFVAIVRVODKDSGLGEIICKLHGHGHFKLQKTYENNYLILTNATLDREKRSEYSLTVIAEDKGTPSLSSVRHFTVQINDINDNPPRF 465

c4 human

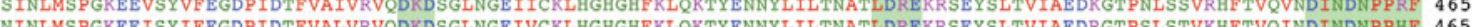
enopus NINLMSTGK-EIAYISEGAPLDTFVALVRVODKDSGLNGE IVVRLHGHGQFKLOKTYENNYLILTNSTLDREKRSEYSLTVIAEDRGVPSLSTVK HFAVQIIDENDNPPRF 471

zebrafish NINLMTOGKEEVAYISEASPVDTFIALVRVDDSDGINGEVVCRLHGHGHFRLOKTYENNYMILTNVSLDREKRSEYSLTVIAEDRGTPSLSTIKHFTVQVQDENDNPPRE 471

mouse QRSRYEFVISENNSPGAYITTVTATDPDLGENGHVTYTILESFVLGSSITTYVTIDPSNGAIYALRIFDHEEVSQITFVVEARDGGSQKQLSSNTTVVLTIIDENDNVPVV 576

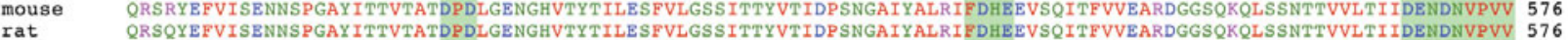
E5 human ORSRYEFVISENNSPGAYITTVTATDPDLGENGOVTYTILESFILGSSITTYVTIDPSNGAIYALRIFDHEEVSOITFVVEARDGGSPKOLVSNTTVVLTIIDENDNVPVV 576 xenopus PTNRYEIVIVENNSPGAHITSVRATDPDQAENGQVTYTILESSIQSSSITTYVTIDPSNGALYALRAFDHEEVNQTMFVVQARDGGVP-QLTTNATVILTVVDENDNAPVI 581

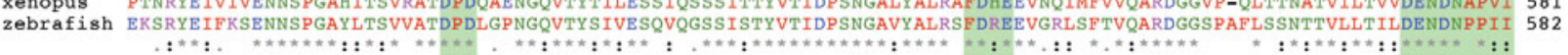

mouse IGPAMHNNTAEISIPKGAESGFHVTRIRVVDRSGANAEFSCSIVSGNEENIFIMDPRSCDIHTNVSMESIPSTEWALSVIIQDKGSPP-LHTKVLLRCMVFDYESVTSTAMTSVSRASLDVS 699 $\begin{array}{lll}\text { mouse } & \text { IGPAMHNNTAEISIPKGAESGF HVTRIRVVDRDSGANAEFSCSIVSGNEENIF IMDPRSCDIHTNVSMESIPSTEWALSVIIQDKGSPP-LHTKVLLRCMVFDYAESVTSTAMTSVSRASLDVS } 699 \\ \text { rat } & \text { VGPAMHNNTADISIPKGVESGFHVTRLRVVDRDSGANAEFTCSIVAGNEENIFIIDPRSCDIHTNVSIDSISSTEWALSVIIQDKGNPP-LHTKVLLRCMVFDYTESVTSTAMTSVSHASLDVS 699 }\end{array}$ IGPAM henopus VVPVLHNNTAEITIPKDAEIGYLVTTIKATDRD GMNSELDCSISAGNEHNIFIMDPRSCEIYTNMSMDIFPNQLLDLHITVQDRGNPP-RTTKAHLRFNILDLIEITEGTPEPYTKQSSMDVS 704 xenopus
zebrafish VAPLISNHTAEVPLWRHAESGHLVTIIKATDRDAGANSELTCSIVGGNDEGLFVIDPRRCELRTNGSVEASGREGEDLAILVODRGASIRLSARAVLHISLRDYPESYSLNPSDINNQSPLDV 704

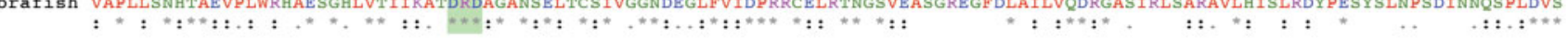

mouse MIIISLGAICAVLLVI 716

rat MIIIISLGAICAVLLVI 716

TM human MIIIISLGICAVLLVI 716

xenopus MIIIISLGAICAVLLVI 721

Sh MilisLGATCGULIV

mouse MVLFATRCNREKKDTR-SYNCRVABSTYQHHPKRPSRQIHKGDITLVPTINGTLPIRSHHRSSPSSSPTLERGOMGSRQSHNSHQSLNSLVTISSNHVPENFSLELTHATPAVE-VSQLL 834 rat MVLFATRCNREKKDTR-SYNCRVAESTYQHHPKRPSRQIHKGDITLVPTINGTLPIRSHHRSSPSSSPTLERGQMGSRQSHNSHQSLNSLVTISSNHVPENFSLELTHATPAVE-VSQLL 834 P human MVLFATRCNREKKDTR-STNCRVAESTYQHHPKRPSRQIHKGDITLVPTINGTLPTRSHHRSSPSSSPTLERGQMGSRQSHNSHQSLNSLVTISSNHVPENFSLELTHATPAVEQVSQLL 835 xenopus MVVFATRCNREKKDNR-SYNCRVAESTYQNHPKRPSRQIHKGDIALVPTLNGTLPIRSHHRSSPSPSPSLERGQMSSRQSQHSRQSLSSLMTISPNHVPENFSLELTHATPAVE-VSQLL 840

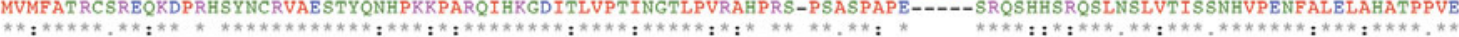

mouse SMLHOGOYOPRPSFRGNKYSSYRYALODMDKFSLKDSGRGDSEAGDSDYDLGRDSPIDRLLGEGFSDLFLTDG--RIPAAMRLCTEECRVLGHSDOCWMPPLPSPSS-DYRSNMFIPGE 951 rat SMLHQGQYQPRPSFRGNKYSRYRYALQDMDKFSLKDSGRGDSBAGDSDYDLGRDSPIDRLLGEGFSDLFLTDG--RIPAAMRLCTEBCRVLGHSDOCWMPPLPSPSS-DYRSNMFIPGE 951 human SMLHOGQY xenopus SMLHQGQYQPRPSFRGNKYSRYRYALQDMDKFSLKDSGRGDSEAGDSDYDLGRDSPIDRLLGEGFSELFLMDGH-RMPPAMRLCTDECRLLGHSDQCWMPPLP SPSS-DYRSNMFIPGE 958 zebrafish --GQYQPRP SFRGNKYRSYRYALNEMDKFSLKDSGRGDSEAGDSDCEMGRESP--LLGEGFSELFTPDGHHRLHPTMKLCTEECRVLGHSDQCWMPPLPSPASSDYRSNLYIPGE 943

mouse EFPAQPQQQHSHQGLD-------DDSQPAENGEKKKSFSTFGKDSPSDEDSGDSS-----TSSLLSEMSSVFQRLLPASLDTFSECNEGDRS--------NSLERRKGPQGKT--GGYPQ 1050 rat EPPAQPQQQHSHPGLD-----DDSQPAESGEKKKSFSTFGKDSPSDEDSGDSS----TSSLLSEMNSVFQRLLPASLDTYSECSEVDRS------NSLERRKGPAQGKT--GGYPQ 1050 human EFPTQPQQQHPHQSLE-------DDAQPADSGEKKKSFSTFGKDSPNDEDTGDTS-----TSSLLSEMSSVFQRLLPPSLDTYSECSEVDRS-------NSLERRKGPLPAKT--VGYPQ 1051 xenopus ELQPQQQPLHNQQQIQAQTPLAEEDMHPVESTEKKKSFSTFGKES-QEEESADTC-----TSSLLSEMSSVFQRLLPPSLDNYTECNEVDRT--------NSLERRKQAPAKA--VSYPQ 1062

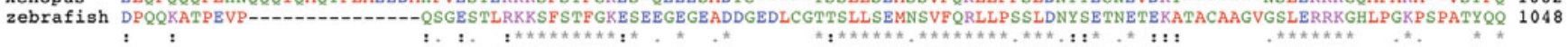

mouse GVAAWAASTHFQNPTNSSGTPLGTHSSVQPS---------SKWLPAMEEIPENYEEDDFDNVLNHLS---DGKHELMDASELVAEINKLLQDVRQS 1134 rat GVAAWAASTHFQNPTSSSGPLGTHSSVQPS--human GVAAWAASTHFONPTTNCGP PLGTHSSVQPS------------SKWLPAMEEIPENYEEDDFDNVLNHLN----DGKHELMDASELVAE INKLLQDVROS 1135 xenopus GVATWAANTHFONPN-NVGTPLGNHSGAQPS------------SKWLPAMEE IPENYEEDEFDNVLNHLN----DGKHELMDASELVAE INKLLQDVRQT 1145

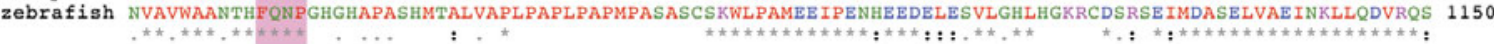

Supplementary Figure S2. Multiple alignment of known pcdh18 proteins. Multiple alignment of mouse (Accession: NP_569715), rat (EDM15003), human (NP_061908), Xenopus (NP_001011150) and zebrafish pcdh18 proteins was performed with ClustalW. S indicates signal sequence; EC1-6, cadherin domains; TM, transmembrane domain; CP, cytoplasmic domain; Green boxes, calcium ion binding sites. The Dab1 binding motif, F/YXNP, is marked with a red box. CM-2 motif conserved among pcdh8, 10, 18, 19 and others is marked with a blue box. 


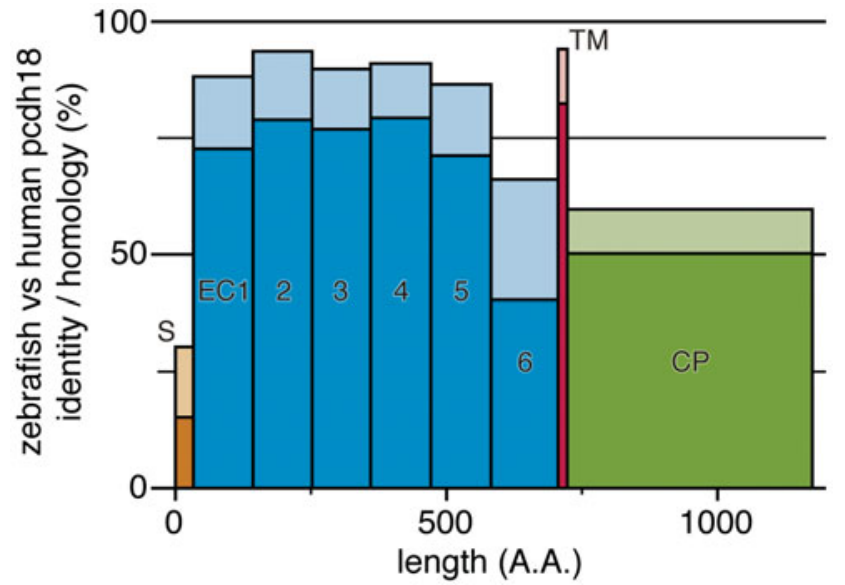

Supplementary Figure S3. Domain structure and identity/homology of zebrafish pcdh18 protein. Bars represent the domains of zebrafish pcdh18 protein. Bar widths indicate protein lengths in number of amino acids (A.A.) and bar heights indicate identity (darkly colored) and homology (lightly colored) of the domain with the human counterpart. Orange bar (S), signal sequence; blue (EC1-6), extracellular cadherin domains 1-6; red (TM), transmembrane domain; green (CP), cytoplasmic domain.
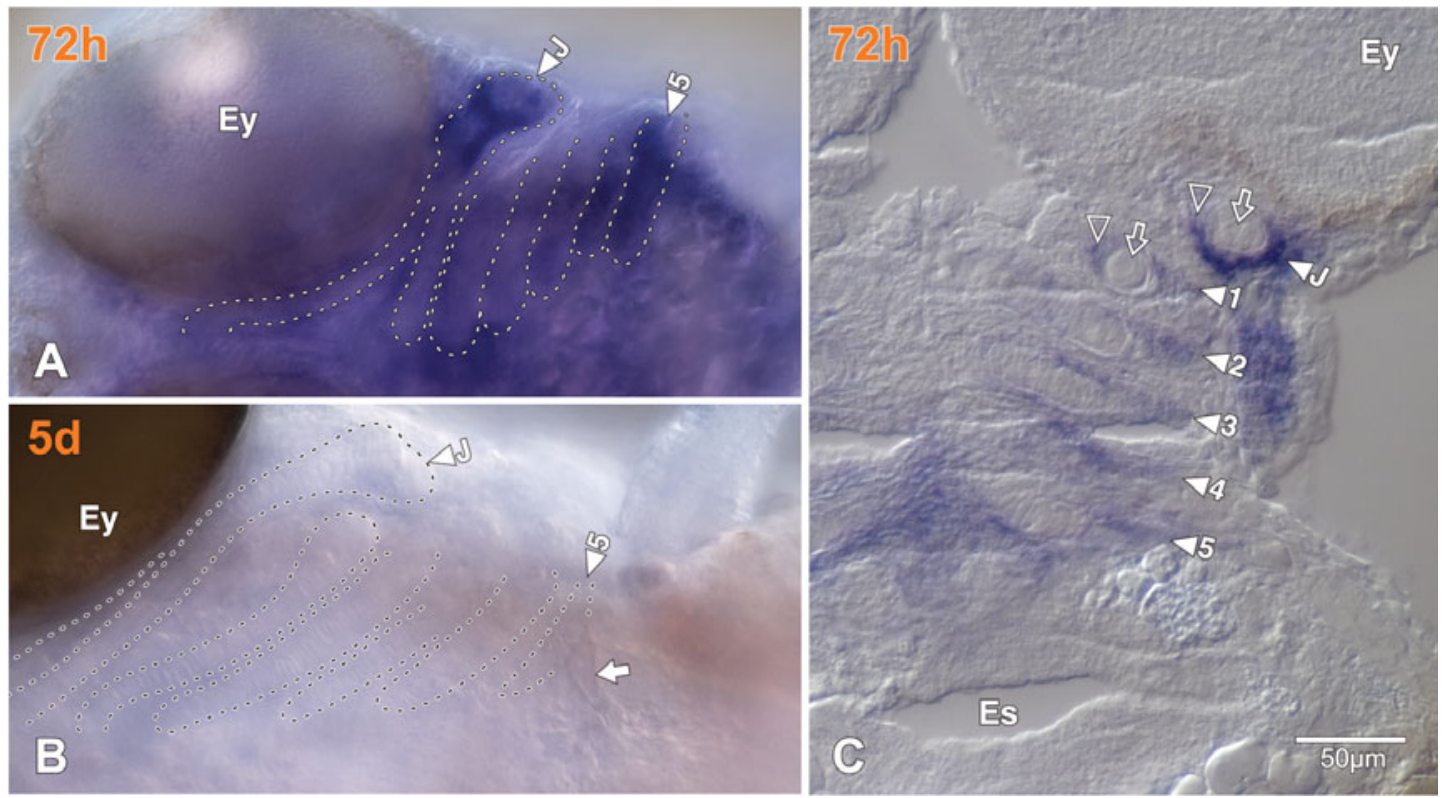

Supplementary Figure S4. pcdh18 expression in the branchial arches. Orange numbers indicate embryonic or larval age post-fertilization. (A,B) Ventral views of embryos at $72 \mathrm{hpf}$ and $5 \mathrm{dpf}$, respectively, with the yolk removed. Rostral to the left, left to the top. Dotted lines outline the jaw and branchial arches. Closed arrowheads identify the arches. (A) The 5th arch where the pharyngeal tooth would attach has diffuse but no tooth-specific pcdh18 staining at 72 hpf. (B) A pharyngeal tooth (closed arrow) appeared by 5 dpf when pcdh18 expression had diminished from the embryo. (C) A coronary cryostat section of a stained 72 hpf embryo cut at the level of the arches (the 4th arch is somewhat off the level of the particular section). The cells expressing pcdh 18 (open arrowheads) surrounds branchial cartilages (open arrows). Abbreviations: 1-5, 1st to 5th branchial arches; Ey, eyes; Es, esophagial lumen, J, jaw arches. 


\section{Related, previously published Int. J. Dev. Biol. articles}

See our recent Special Issue Ear Development edited by Fernando Giraldez and Bernd Fritzsch at: http://www.ijdb.ehu.es/web/contents.php?vol=51\&issue=6-7

See our Special Issue Developmental Morphodynamics edited by Lev Beloussov and Richard Gordon at: http://www.ijdb.ehu.es/web/contents.php?vol=50\&issue=2-3

Cadherin-6 is required for zebrafish nephrogenesis during early development Fumitaka Kubota, Tohru Murakami, Kenji Mogi and Hiroshi Yorifuji Int. J. Dev. Biol. (2007) 51: 123-129

Fate of cranial neural crest cells during craniofacial development in endothelin-A receptor-deficient mice Makoto Abe, Louis-Bruno Ruest and David E. Clouthier Int. J. Dev. Biol. (2007) 51: 97-105

TBX1, a DiGeorge syndrome candidate gene, is inhibited by retinoic acid Lifeng Zhang, Tao Zhong, Yuexiang Wang, Qiu Jiang, Houyan Song and Yonghao Gui Int. J. Dev. Biol. (2006) 50: 55-61

Fibroblast growth factor signalling and regional specification of the pharyngeal ectoderm

Nina Trokovic, Ras Trokovic and Juha Partanen

Int. J. Dev. Biol. (2005) 49: 797-805

Common mechanisms for boundary formation in somitogenesis and brain development: shaping the 'chic' chick Yoshiko Takahashi

Int. J. Dev. Biol. (2005) 49: 221-230

NK-2 class homeobox genes and pharyngeal/oral patterning: Nkx2-3 is required for salivary gland and tooth morphogenesis. Christine Biben, Cheng-Chun Wang and Richard P Harvey Int. J. Dev. Biol. (2002) 46: 415-422

Hoxb-5 is expressed in gill arch 5 during pharyngeal arch development of flounder Paralichthys olivaceus embryos. T Suzuki, A S Srivastava and T Kurokawa Int. J. Dev. Biol. (1999) 43: 357-359

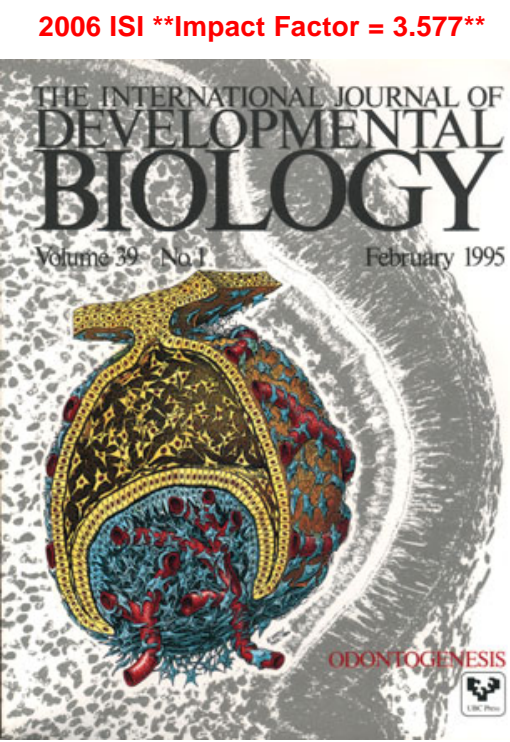

